\title{
A randomized comparative trial of corticosteroid phonophoresis, local corticosteroid injection, and low-level laser in the treatment of carpal tunnel syndrome
}

\author{
Mahsa Asheghan, M.D. ${ }^{1},{ }^{*}$ Amidoddin Khatibi Aghda, M.D. ${ }^{2}$, Vahid Sobhani, M.D. ${ }^{3}$, \\ Seyed Ebrahim Hashemi, M.D. ${ }^{1}$, Mohammad Taghi Hollisaz, M.D. ${ }^{1}$ \\ 1: Baqiyatallah University of Medical Sciences, School of Medicine, \\ Department of Physical Medicine and Rehabilitation \\ 2: Shabid Sadoughi University of Medical Sciences, School of Medicine, \\ Department of Physical Medicine and Rehabilitation \\ 3: Baqiyatallah University of Medical Sciences, School of Medicine, \\ Institute of life style, exercise physiology research center
}

\begin{abstract}
Background and aims: Carpal tunnel syndrome is the most prevalent peripheral neuropathy and has a considerable burden on health services. We tried to compare the therapeutic effects of local corticosteroid injection, low-level laser, and corticosteroid phonophoresis in the treatment of carpal tunnel syndrome. Subjects and methods: We performed a randomized clinical trial with three parallel groups. The study was carried out at a University Hospital. In total, 42 participants including 31(73.8\%) women were randomly allocated to the treatment groups with equal sizes. We assessed pain, symptom severity and functional status with Boston Carpal Tunnel Questionnaire, and performed median nerve conduction velocity studies. Evaluations were done before the interventions and in the fourth week of study. For the group corticosteroid, under the guidance of sonography, methylprednisolone with lidocaine was injected into the carpal tunnel. For laser therapy, we administered 10 sessions, each lasting 10 seconds. We used topical hydrocortisone acetate gel $10 \%$ as the anti-inflammatory agent with phonophoresis, 3 times per week for 10 sessions. Results: Within-group analyses with paired t-test showed that local corticosteroid, laser, and phonophoresis are all effective treatments. Between-group analyses with ANOVA indicated that there were significant differences among the groups after four weeks in terms of pain $(p=0.004)$, in favor of corticosteroid; and in sensory delay ( $\mathrm{p}=0.001)$, in favor of laser. For the Boston Carpal Tunnel Questionnaire and median nerve motor latency, the results were not significant. There was no important side-effect after four weeks of follow-up.

Conclusion: The three treatments are comparable and beneficial for carpal tunnel syndrome.
\end{abstract}

Key words: Carpal Tunnel Syndrome $\cdot$ Median nerve $\cdot$ Corticosteroid injection $\cdot$ Laser therapy $\cdot$ Corticosteroid phonophoresis

\section{Introduction}

Carpal tunnel syndrome (CTS) is the most prevalent peripheral neuropathy ${ }^{11}$. The disease leads to sensory and motor problems, and the subsequent disability and eco-

*Addressee for Correspondence:

Amidoddin Khatibi Aghda, Department of Physical Medicine and Rehabilitation, School of Medicine, Shahid Sadoughi University of Medical Sciences. Hesabi St, Yazd, Islamic Republic of Iran, Postal code: 8915863943

Tel: (+98) 3538282769 / Fax: (+98) 3538282767 /

E-mail: a.khatibi@ssu.ac.ir nomic impact of work absence are significant ${ }^{2)}$. CTS still has a considerable burden on health services. The goal of management is to decrease the pressure on the median nerve. Any measure that reduces the swelling in the carpal tunnel may serve as a treatment modality. Systematic reviews using Delphi strategy on the effectiveness of surgical and nonsurgical interventions were conducted to find consensus on an efficient therapeutic program. As

Received date: July 31th, 2019

Accepted date: Octobar 28th, 2019

J-STAGE Advance Publication Date: April,18th 2020 
stated in the European Hand guide, the physician should consider the severity and duration of the symptoms, and previous treatments, for selecting the best therapeutic strategy. Beside of patients' education; splinting, corticosteroid injection (CSI), corticosteroid injections plus splinting, and surgery is suggested for treatments of CTS ${ }^{3)}$.

A systematic review of randomized trials showed that heterogeneity in the assessment of therapeutic outcomes does not allow affirming a universal strategy for the treatment of CTS ${ }^{4}$. Controversies can be recognized even around established treatment methods like CSI ${ }^{5-8}$. There are therapeutic modalities for CTS that are not specified in the guidelines. Among the possible noninvasive treatments of CTS, low-intensity laser therapy (LLT) and phonophoresis therapy (PPT) are two choices.

LLT includes the application of a read or near-infrared laser beam. The wavelength of the beam can be set in a range from 600 to $1070 \mathrm{~nm}$ with the power of 1 to $1000 \mathrm{~mW}$. The setup parameters of the laser are categorized into two groups of irradiation, and treatment parameters. The parameters can affect the outcomes of the treatment. Some researchers showed strong evidence in favor of LLT in pain control for the short-term ${ }^{9,10)}$. However, there are others who did not find obvious benefit for LLT in the treatment of CTS ${ }^{2,11)}$.

Instead of an injection, PPT provides a safe and painless route of administrating anti-inflammatory medications, by using ultrasound. Of anti-inflammatory medicines, corticosteroid and non-steroidal anti-inflammatory drugs have been used in PPT. In a study on the application of PPT with a corticosteroid, dexamethasone sodium phosphate was successfully used for the treatment of CTS 12). There is a relative paucity of information on the effects of PPT in treating CTS. Some studies indicated desirable therapeutic outcomes after PPT ${ }^{12}$. There are several reports suggesting the application of PPT as an adjuvant to usual conservative treatments ${ }^{13-15)}$

According to the literature, evaluation of LLT and PPT, and comparing their therapeutic effects with other conservative treatments is still warranted. Meta-analyses, systematic reviews and Delphi questionnaire, though valuable, would not replace trials when there are knowledge gaps regarding relatively more recent treatment techniques. Until reliable information about different therapeutic methods is collected, it is difficult to design the best intervention strategy.

We conducted a trial to compare the effects of CSI, LLT, and PPT in symptom control and median nerve conduction velocity. Our hypothesis was that the treatments would be different in providing symptomatic relief and electrophysiological improvement for patients in primary care.

\section{Subjects and methods}

\section{Design and Setting}

We performed a randomized clinical trial with three parallel groups. The study began in March 2017 and ended in August 2017. Our setting was an outpatient clinic of physical medicine and rehabilitation at the Baqiyatallah University Hospital. The Hospital is a large referral subspecialty center in Tehran.

\section{Recruitment}

Patients were recruited from the waiting list of the hospital clinic of the department of physical medicine and rehabilitation. Some of them have come to the clinic due to the manifestations of CTS, and the others have been referred by other physicians for the diagnostic workup of CTS. Potential participants were invited to attend a visit for eligibility. Eligibility was confirmed by a resident of physical medicine and rehabilitation who interviewed patients, filled in a questionnaire on past medical history and the risk factors for CTS and performed detailed physical examinations. Then, one of the authors confirmed the diagnosis of CTS. Enrolled patients who gave consent were immediately allocated randomly to one of the treatment groups. Before any treatment, participants filled the Boston Carpal Tunnel Questionnaire (BCTQ) and underwent electrodiagnostic studies.

Beside of history and general physical examination, any relevant sensory and motor symptoms with their exact characteristics and patterns were recorded. Presence of Tinel's sign, the result of Phalen's maneuver, and the strength of the abductor pollicis brevis were assessed by muscle manual testing, and range of motion for digits and the wrist measured for flexion and extension. For several complicated diagnoses, a committee of the authors made a decision.

In order to provide a more homogenous sample, we confined our sample to patients with mild to moderate idiopathic and occupational CTS. Participants with diabetes mellitus (types I and II), hypothyroidism, arthritis, and pregnancy were excluded. Participants with a body mass index of more than $30 \mathrm{~kg} / \mathrm{m}^{2}$ did not enter the study. Patients with trauma as the probable cause of CTS or patients who had undergone CSI, LLT, or PPT within the previous 6 months of the study were excluded. We also evaluated the participants for the differential diagnoses of CTS including polyneuropathy, radiculopathy, motor neuron disease, spondylotic myelopathy, syringomyelia, and multiple sclerosis.

Only one hand from each individual was selected for the analytic sample. In patients with bilateral symptoms, we included one hand randomly by tossing a coin, and in case both hands were not equally symptomatic the dominant hand was included. 


\section{Outcome measures}

One examiner blinded to the study question performed nerve conduction velocity studies. The pain was the main outcome measure and was assessed in a visual analog scale. Bilateral median sensory and motor median nerve conduction studies were carried out at the level of the wrist by a 3 channel electromyography device (Synergy Ultrapro S100, Natus, USA). The assessments were done according to the American Association of Electrodiagnostic Medicine (AAEM) guidelines ${ }^{16)}$. All assessments were done at the room temperature of $25^{\circ} \mathrm{C}$ and the skin temperature of $32-33^{\circ} \mathrm{C}$. The ground electrode was placed on the dorsum of the hand. For motor nerve conduction study, surface electrodes were located over abductor pollicis brevis. The Stimulation was done at $8 \mathrm{~cm}$ proximal to the active electrode. The sensory responses were obtained at the third digit, stimulating antidromically at 14 $\mathrm{cm}$. Motor latency less than $4.1 \mathrm{~ms}$ and sensory latency less than $3.6 \mathrm{~ms}$ were considered as the normal values. We used a bandwidth setting of $20 \mathrm{~Hz}$ to $3 \mathrm{kHz}$, sweep speed of 1 millisecond/division and gain of 10 to $20 \mu \mathrm{V} /$ $\mathrm{cm}$.

The BCTQ was used for the assessment of clinical outcomes ${ }^{17,18)}$. The questionnaire is a patient-based outcome measure with two distinct scales. The Symptom Severity Scale (SSS) has 11 questions and the Functional Status Scale (FSS) includes 8 items. The questions use five-point rating scales. The sum of the scores divided by the number of items yields the final disability score (1 to 5) of the patient. A higher score shows greater disability. We assessed the severity of the syndrome according to a standard method.

\section{Study interventions}

For the group CSI we injected $40 \mathrm{mg}$ Methylprednisolone mixed with $10 \mathrm{mg}$ lidocaine. Under the guidance of sonography (The 25-Mylab ultrasound system with a 10-18 $\mathrm{MHz}$ multi-D linear array transducer, Esaote, Toscana, Italy), a 25-gauge needle was inserted $1 \mathrm{~cm}$ proximal to the wrist-flexion crease just on the palmaris longus tendon. The mixture was slowly administered into the carpal tunnel. Participants were instructed to have minimum wrist movement within 24 hours of injection. Also, they were informed on how to use an icepack or non-steroidal inflammatory drug, if needed. The injection was repeated for every two weeks, and in total, 3 injections were administered for each patient.

For LLT we administered a low potent continuous mode laser (multilevel locked system indium laser of M1 type with the trademark of ASA, a company of ELEN group), characterized by the amplitude of $780 \mathrm{~nm}$, frequency of $6500 \mathrm{HZ}$, the wavelength of $880 \mathrm{~nm}$, and intensity of $20 \mathrm{~J} / \mathrm{cm}^{2}$. We repeated laser therapy every 3 days for 4 weeks. Overall, we performed 10 sessions of
LLT, each lasting 10 seconds, on 5 locations along the median nerve passage above the carpal tunnel.

PPT was administered to the area over the carpal tunnel for 5 minutes each session, 3 times per week for 10 sessions, with the frequency of $1 \mathrm{MHz}$, the intensity of $1 \mathrm{~W} / \mathrm{cm}^{2}$, and the transducer area of $5 \mathrm{~cm}^{2}$. We used topical ointment of hydrocortisone as the anti-inflammatory agent. Participants were instructed not to use other local or systemic analgesic medications except for acetaminophen p.r.n. in severe pain.

\section{Ethical considerations}

Our research was conducted according to the declaration of Helsinki. Ethics approval was obtained from the institutional review boards of the University of Baqiyatallah. All participants gave signed written consents. They received information on the rationale of the research.

\section{Randomization and statistical analyses}

Random allocation was centrally performed with blocked randomization. Random numbers were generated by a computer. We provided three samples, each including 14 participants. Data are presented as mean (standard deviation) for continuous and as numbers (proportions) for categorical variables. Data were checked for normality using the Kolmogorov-Smirnov test. Differences in continuous variables were compared using one-way ANOVA. For within-group analyses, we used paired t-test to compare the outcomes at baseline and after the treatments. All data analyses were performed with IBM SPSS version 20 for windows. We considered a two-tailed p-value of less than 0.05 as statistically significant.

\section{Results}

In total, we had 42 participants including $31(73.8 \%)$ women. They were randomly allocated to three equalsize groups of CSI, LLT, and PPT. Figure 1 shows patients flow during the study, and Table 1 shows demographic data of the sample. Of the participants, 36 (85.7\%) were right-handed. The chi-squared test showed that was no significant correlation between hand dominancy and the side of CTS $(\mathrm{p}=0.423)$. Table 2 shows the results of measurements at the beginning and at the end of the study. ANOVA test showed that at the beginning of the study the three groups were similar in term of subjective pain $(\mathrm{p}=0.130)$.

Between-group analyses using ANOVA test showed that there were significant differences among the groups after four weeks in terms of pain ( $p=0.004)$, and sensory latency ( $\mathrm{p}=0.001$ ) scores. However, in motor latency, and BCTQ scores there was no significant difference between the groups $(\mathrm{p}=0.160$ and 0.071 , respectively). Post hoc test with Bonferroni adjustment showed that for pain there was a statistically significant 
difference between CSI and LLT with the superiority of CSI ( $p=0.003)$. There was no significant difference in pain score between CSI and PPT ( $=0.898)$ and between PPT and LLT $(p=0.055)$. For sensory latency, there was a significant difference between LLT and PPT in favor of LLT ( $p=0.001)$. Other comparisons were not significant (all $p \geq 0.148$ ). None of the participants reported significant side-effect after four weeks of intervention.

\section{Discussion}

We tried to compare three different therapeutic modalities: CSI, LLT, and PPT for the treatment of CTS in primary care. Our study showed that after four weeks, the three treatment programs were effective in relieving pain and improving electrodiagnostic features. While there were some statistically significant differences between the groups in terms of pain and sensory latency, our evidence failed to show a marked clinically important difference for all the outcomes when the three strategies com- pared with each other. Overall, our results are similar to those of other previously published research. Research on a variety of therapeutic measures has been indicated that within the short term, there is no treatment that could be selected as the best.

A systematic review of randomized controlled trials indicated that there is strong evidence on desirable clinical outcomes of LLT for CTS ${ }^{9}$. But as a general statement, the efficacy remains high for 5 weeks. Thereafter, the strength of evidence decreases in time to even no evidence at three months follow-up. Limited evidence exists for treatment outcomes in favor of LLT at 6 months versus placebo. In another review on nine randomized controlled trials, the effectiveness of LLT on reducing pain, improving hand function, and increasing grip strength among patients with CTS was investigated ${ }^{2)}$. It was reported that despite heterogeneity in terms of laser parameters there was no strong evidence supporting LLT. However, there was a clinically significant trend for beneficial outcomes in the short term. In a comparative trial, LLT

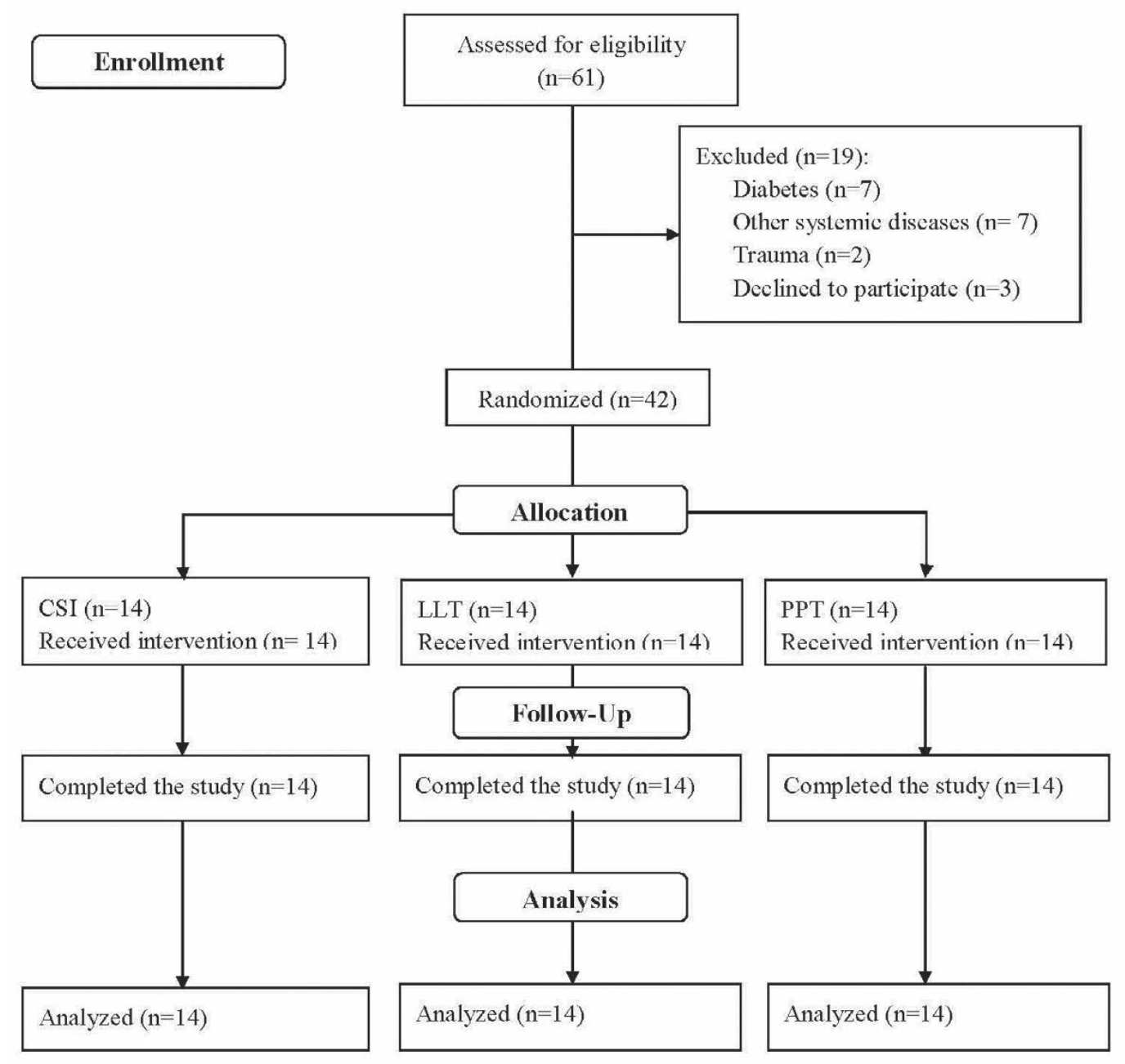

Figure 1: Participants flow diagram (CSI: corticosteroid injection, LLT: low-level laser therapy, PPT: phonophoresis therapy) 
was compared to ultrasound in the treatment of CTS among patients with diabetic neuropathy ${ }^{10}$. The results showed that both treatments are effective for patients with mild to moderate CTS. A meta-analysis showed that in general, the evidence does not support LLT regarding except for some outcomes like grip strength ${ }^{11)}$. Meanwhile, the study warranted further well-designed trials with long follow-ups to establish LLT efficacy. We found much controversy around the application of LLT in the literature. However, our study showed short term benefits for LLT in the treatment of mild and moderate CTS. We recognized desirable outcomes of LLT regarding pain, the severity of symptoms and function (BCTQ), and electrodiagnostic features in our patients. It seems that until LTT is standardized, it is difficult to accumulate strong evidence to support or oppose its efficacy. It should be emphasized that to our knowledge there is no published study to compare the efficacy of LLT and PPT.

In a study, some researchers compared the efficacy of several treatment programs for CTS including PPT with non-steroid anti-inflammatory drugs, and CSI ${ }^{13}$. Participants were examined before the interventions and three months later with electrophysiological assessments, and sensory and motor evaluations. Functional disability was assessed using the Duruoz hand index. For group local corticosteroid $(n=23)$, betamethasone dipropionate 0.5 mg was injected into the carpal tunnel. They had two groups of PPT, one of them used a non-steroidal anti-inflammatory drug $(n=20)$, and the other used betamethasone valerate $0.1 \%(n=22)$ as the medication. Fifteen sessions of PPT were carried out for participants. While there were differences in some items of the outcome, the study did not indicate a generic superior treatment. They noted that splinting the wrist in the neutral position would not lead to remarkable improvement, but CSI and PPT showed clear benefits in the treatment of CTS. The eligibility criteria were similar to ours. We followed our patients for one month; however, the results were almost the same. Our data indicated that PPT would be an efficient alternative to CSI. Mean difference in pain control

Table 1: Demographic data of the groups

\begin{tabular}{lccc}
\hline & \multicolumn{3}{c}{ Groups } \\
\cline { 2 - 4 } & CSI $(\mathrm{n}=14)$ & LLT $(\mathrm{n}=14)$ & PPT $(\mathrm{n}=14)$ \\
\hline Female & 11 & 9 & 11 \\
\hline Age (year) & $48.6(11.6)$ & $49.4(5.2)$ & $52.4(3.8)$ \\
\hline Weight $(\mathrm{kg})$ & $78.3(9.6)$ & $80.1(11.8)$ & $77.8(9.3)$ \\
\hline
\end{tabular}

Table 2: Within-group analyses of the results before the intervention and after four weeks of follow-up

\begin{tabular}{|c|c|c|c|c|c|}
\hline & & & \multicolumn{3}{|c|}{ Group } \\
\hline & & & CSI $(n=14)$ & LLT $(n=14)$ & PPT $(n=14)$ \\
\hline \multirow{16}{*}{ Score Mean (SD) } & \multirow{4}{*}{ PainBefore intervention } & $6.7(2.3)$ & $8.0(1.7)$ & $7.3(2.0)$ & \\
\hline & & 4 weeks & $1.8(0.9)$ & $3.7(2.3)$ & $2.7(1.0)$ \\
\hline & & Difference & $4.9(2.1)$ & $4.3(2.0)$ & $5.3(1.9)$ \\
\hline & & p value & $<0.001$ & $<0.001$ & $<0.001$ \\
\hline & \multirow{4}{*}{ BCTQ } & Before intervention & $31.2(7.3)$ & $34.4(9.2)$ & $30.7(7.3)$ \\
\hline & & 4 weeks & $15.2(4.4)$ & $22.9(7.0)$ & $20.0(1.9)$ \\
\hline & & Difference & $16.0(6.4)$ & $11.5(6.3)$ & $10.9(5.9)$ \\
\hline & & $\mathrm{p}$ value & $<0.001$ & 0.002 & $<0.001$ \\
\hline & \multirow{4}{*}{$\begin{array}{l}\text { Median Motor latency } \\
\text { (millisecond) }\end{array}$} & Before intervention & $4.7(0.6)$ & $4.3(0.6)$ & $4.5(0.4)$ \\
\hline & & 4 weeks & $4.2(0.5)$ & $3.8(0.4)$ & $4.1(0.3)$ \\
\hline & & Difference & $0.5(0.3)$ & $0.4(0.6)$ & $0.4(0.4)$ \\
\hline & & $\mathrm{p}$ value & 0.045 & 0.012 & $<0.001$ \\
\hline & \multirow{4}{*}{$\begin{array}{l}\text { Median Sensory latency } \\
\text { (millisecond) }\end{array}$} & Before intervention & $4.8(0.6)$ & $4.1(0.3)$ & $4.4(0.4)$ \\
\hline & & 4 weeks & $4.1(0.5)$ & $3.6(0.3)$ & $3.9(0.3)$ \\
\hline & & Difference & $0.6(0.3)$ & $0.5(0.2)$ & $0.5(0.3)$ \\
\hline & & p value & 0.140 & 0.003 & 0.006 \\
\hline
\end{tabular}


was the highest for PPT. We thought that the participants were more satisfied with PPT. Maybe the patients are more comfortable with the route of administration in PPT.

In a randomized controlled trial, PPT and iontophoresis of corticosteroid in conjunction with wrist splinting were compared in efficacy for treatment of CTS ${ }^{14)}$. Symptom severity, motor skills, and hand function were assessed with appropriate instruments. Participants were examined at the beginning of the study and in the third month after the intervention. Betamethasone was used with 3 weeks of PPT $(n=18)$ and 3 weeks of iontophoresis $(n=16)$; and the control group received splinting only $(n=18)$. The study indicated that severity of symptoms decreased at the end of study (all p values $\leq 0.001$ ) in the 3 groups; while grip strength and hand function did not indicate significant improvement. There was no report on electrophysiology at the end of the study. Of course, with respect to the severity of symptoms, PPT showed favorable result compared to control. The study concluded that PPT with splinting would be efficient for relieving symptoms. The sample size in that study is comparable to ours. We examined the functional status and severity of symptoms with BCTQ. Our study showed that CSI, LLT, and PPT cause a favorable reduction in BCTQ scores. Pain measurements indicated desirable reduction among all of our intervention groups, too.Besides, we followed our patients with electrodiagnostic study in which desirable changes were seen for all groups.

Our study was the first one to compare the efficacy of LLT and PPT. The participants in our study were compliant, and the research team was expert. Meanwhile, we did not evaluate the long term effects of the treatments. Larger research with prolonged follow-up periods is required to investigate the effects of combined treatments. Also, our research implied that there is some therapeutic potential in PPT to be investigated. Future studies should include randomized controlled trials with large sample size to assess the place of PPT in the clinic.

\section{Conclusion}

Patients with CTS would benefit from CSI, LLT, or PPT, at least in the short term. Specifically, PPT merit to be considered as a first-line in the treatment of CTS, because of pain relief and ease of administration.

\section{References}

1: Ren, Y.M., X.S. Wang, Z.J. Wei, B.Y. Fan, W. Lin, et al. Efficacy, safety, and cost of surgical versus nonsurgical treatment for carpal tunnel syndrome: A systematic review and meta-analysis. Medicine (Baltimore). 2016; 95: p. e4857.

2: Burger, M., R. Kriel, A. Damon, A. Abel, A. Bansda, et al. The effectiveness of low-level laser therapy on pain, self-reported hand function, and grip strength compared to placebo or "sham" treatment for adults with carpal tunnel syndrome: A systematic review. Physiother Theory Pract. 2017; 33: p. 184-197.

3: Huisstede, B.M., J. Friden, J.H. Coert, P. Hoogvliet, and H.G. European. Carpal tunnel syndrome: hand surgeons, hand therapists, and physical medicine and rehabilitation physicians agree on a multidisciplinary treatment guideline-results from the European HANDGUIDE Study. Arch Phys Med Rehabil. 2014; 95: p. 2253-63.

4: Huisstede, B.M.A., P. Hoogvliet, T.P.C. Franke, M.S. Randsdorp, and B.W. Koes. Carpal Tunnel Syndrome: Effectiveness of Physical Therapy and Electrophysical Modalities. An Updated Systematic Review of Randomized Controlled Trials. Arch Phys Med Rehabil. 2017.

5: Kaile, E. and J.D.P. Bland. Safety of corticosteroid injection for carpal tunnel syndrome. J Hand Surg Eur Vol. 2017: p. 1753193417734426.

6: Guo, X.Y., M.X. Xiong, Y. Zhao, F.D. He, X.Q. Cheng, et al. Comparison of the Clinical Effectiveness of Ultrasound-Guided Corticosteroid Injection with and without Needle Release of the Transverse Carpal Ligament in Carpal Tunnel Syndrome. Eur Neurol. 2017; 78: p. 33-40.

7: Evers, S., A.J. Bryan, T.L. Sanders, T. Gunderson, R. Gelfman, et al. Corticosteroid Injections for Carpal Tunnel Syndrome:
Long-Term Follow-Up in a Population-Based Cohort. Plast Reconstr Surg. 2017; 140: p. 338-347.

8: Babaei-Ghazani, A., P. Roomizadeh, B. Forogh, S.M. Moeini-Taba, A. Abedini, et al. Ultrasound-Guided Versus Landmark-Guided Local Corticosteroid Injection for Carpal Tunnel Syndrome: A Systematic Review and Meta-Analysis of Randomized Controlled Trials. Arch Phys Med Rehabil. 2017.

9: Franke, T.P., B.W. Koes, S.J. Geelen, and B.M. Huisstede. Do Patients With Carpal Tunnel Syndrome Benefit From Low-Level Laser Therapy? A Systematic Review of Randomized Controlled Trials. Arch Phys Med Rehabil. 2017.

10: Ahmed, O.F., A.M. Elkharbotly, N. Taha, and A.B. Bekheet Treatment of mild to moderate carpal tunnel syndrome in patients with diabetic neuropathy using low level laser therapy versus ultrasound controlled comparative study. BBA Clin. 2017; 8: p. 43-47.

11: Bekhet, A.H., B. Ragab, A.I. Abushouk, A. Elgebaly, and O.I. Ali. Efficacy of low-level laser therapy in carpal tunnel syndrome management: a systematic review and meta-analysis. Lasers Med Sci. 2017; 32: p. 1439-1448.

12: Bakhtiary, A.H., E. Fatemi, M. Emami, and M. Malek. Phonophoresis of dexamethasone sodium phosphate may manage pain and symptoms of patients with carpal tunnel syndrome. Clin J Pain. 2013; 29: p. 348-53.

13: Soyupek, F., S. Kutluhan, G. Uslusoy, E. Ilgun, S. Eris, et al. The efficacy of phonophoresis on electrophysiological studies of the patients with carpal tunnel syndrome. Rheumatol Int. 2012; 32: p. 3235-42.

14: Gurcay, E., E. Unlu, A.G. Gurcay, R. Tuncay, and A. Cakci. Assessment of phonophoresis and iontophoresis in the treatment of carpal tunnel syndrome: a randomized con- 
trolled trial. Rheumatol Int. 2012; 32: p. 717-22.

15: Yildiz, N., N.S. Atalay, G.O. Gungen, E. Sanal, N. Akkaya, et al. Comparison of ultrasound and ketoprofen phonophoresis in the treatment of carpal tunnel syndrome. J Back Musculoskelet Rehabil. 2011; 24: p. 39-47.

16: Jablecki, C.K., M.T. Andary, M.K. Floeter, R.G. Miller, C.A. Quartly, et al. Practice parameter: Electrodiagnostic studies in carpal tunnel syndrome. Report of the American Association of Electrodiagnostic Medicine, American Academy of Neurology, and the American Academy of Physical Medicine and Rehabilitation. Neurology. 2002; 58: p. 1589-92.

17: Leite, J.C., C. Jerosch-Herold, and F. Song. A systematic review of the psychometric properties of the Boston Carpal Tunnel Questionnaire. BMC Musculoskelet Disord. 2006; 7: p. 78.

18: Greenslade, J.R., R.L. Mehta, P. Belward, and D.J. Warwick. Dash and Boston questionnaire assessment of carpal tunnel syndrome outcome: what is the responsiveness of an outcome questionnaire? J Hand Surg Br. 2004; 29: p. 159-64.

\section{Competing interests}

The authors declare that they have no competing interests.

\section{Funding}

This research received no specific grant from any funding agency in the public, commercial, or not-for-profit sectors. 